\title{
Behavioral healthy nutrition and physical activity counseling in cardiovascular disease prevention: where we are now?
}

\author{
Demosthenes B. Panagiotakos ${ }^{1,2,3,4}$, Matina Kouvari ${ }^{1}$ \\ ${ }^{1}$ Department of Nutrition and Dietetics, School of Health Science and Education, Harokopio University, Athens, Greece; ${ }^{2}$ Department of \\ Kinesiology and Health, School of Arts and Sciences, Rutgers University, New Brunswick, NJ, USA; ${ }^{3}$ Faculty of Health, University of Canberra, \\ Canberra, Australia; ${ }^{4}$ School of Allied Health, College of Science, Health and Engineering, La Trobe University, Melbourne, Australia \\ Correspondence to: Prof. Demosthenes B. Panagiotakos, FRSPH, FACE. Department of Nutrition-Dietetics, School of Health Science and Education, \\ Harokopio University, 70 Eleftheriou Venizelou (Thiseos) St. 176 71, Kallithea, Greece. Email: d.b.panagiotakos@usa.net. \\ Provenance: This is an invited Editorial commissioned by Editor-in-Chief Yilei Mao (Department of Liver Surgery, Peking Union Medical College \\ Hospital, Chinese Academy of Medical Sciences, Beijing, China). \\ Comment on: Patnode CD, Evans CV, Senger CA, et al. Behavioral Counseling to Promote a Healthful Diet and Physical Activity for Cardiovascular \\ Disease Prevention in Adults Without Known Cardiovascular Disease Risk Factors: Updated Evidence Report and Systematic Review for the US \\ Preventive Services Task Force. JAMA 2017;318:175-93.
}

Submitted Sep 22, 2017. Accepted for publication Apr 10, 2018.

doi: 10.21037/hbsn.2018.11.16

View this article at: http://dx.doi.org/10.21037/hbsn.2018.11.16

The decline in age-adjusted mortality rates attributed to cardiovascular disease (CVD) has been characterized as the success story of the past four decades, worldwide. In the United States, a similar downtick has been recorded since 1978. The rapid progress in the fields of prevention and management may be responsible for this promising landscape in the CVD spectrum (1). Nevertheless, CVD still remains the largest single contributor to global mortality with up to date evidence indicating abatement in the rate of the aforementioned mortality decline (1). In the United States, CVDs are on the top of the disability rank possessing a large economic burden for the public health system; the total direct medical costs between 2012 and 2030 are projected to increase from $\$ 396$ to $\$ 918$ billion (2).

As clearly demonstrated by the Non-Communicable Diseases Global Monitoring Framework, effective primary prevention strategies are demanded to meet these challenges. In 2012, the American Heart Association revealed a set of Strategic Impact Goals targeting "by 2020, to improve the cardiovascular health of all Americans by $20 \%$, while reducing deaths from CVDs and stroke by 20\%" (2). That is when a new term was introduced to address this target, "the ideal cardiovascular health"; defined as the absence of CVD manifestation with the simultaneous presence of optimal levels in seven lifestyle and clinical metrics (i.e.,
Life's Simple 7) (2). The vast majority of Americans is far from meeting the current nutritional and physical activity recommendations while they present a high prevalence in major CVD risk factors including abnormal lipid and glucose profile, increased blood pressure and abdominal obesity (3). Findings from the INTERHEART large scale case-control study suggested that these modifiable risk factors accounted for more than $90 \%$ of the myocardial infarction risk (4); at the same time, there is unambiguous evidence that prudent dietary habits and physically active lifestyle are inversely associated with the aforementioned clinical manifestations (5-7).

Following the need for well-documented low-cost practices in the primary CVD prevention spectrum, an updated evidence report and systematic review was published in $\mathcal{F} A M A$ by the US Preventive Services Task Force (USPSTF) accompanied by a recommendation statement guideline for primary health care professionals. The authors conducted a systematic review with the hitherto randomized clinical trials (RCTs) examined behavioral counseling (i.e., healthy diet, increased physical activity, decreased sedentary time or a combination of these) applied to non-obese adults without known hypertension, dyslipidemia, and diabetes/prediabetes. Four distinctive outcomes in relation to intervention were discussed; CVD 
morbidity and/or mortality (i.e., direct outcomes), clinical CVD risk factors (i.e., intermediate outcomes), lifestyle modifications (i.e., intervention efficiency) and potential harmful effects. Eighty-eight RCTs met the inclusion criteria, most of which were conducted in the United States with a 6- to 12-month follow-up. An overall improvement in dietary habits and physical activity status was observed after the intervention. However, it is important to mention that in case of sparse trials with longer follow-up period the effect of dietary counseling was attenuated whilst as for physical activity counseling, results were more favorable in RCTs where all participants had a suboptimal physical activity level at baseline. Regarding the intermediate cardiac health outcomes, the pooled analysis highlighted a significant yet slight or modest improvement in blood pressure, blood lipids profile (i.e., low density lipoprotein, total cholesterol) and adiposity measurements (i.e., body mass index, waist circumference). Most importantly, a doseresponse association was suggested by the authors with the aforementioned outcomes being more evident ranking from low- to medium- and high-intensity interventions. The available findings regarding the independent-of-physicalactivity sedentary behavior were scarce and generally less consistent. Besides the overall beneficial effect of behavioral counseling on intermediate cardiac health outcomes and lifestyle modification, no statistically and most importantly, clinically significant outcomes were observed regarding CVD morbidity, mortality and health related quality of life. As speculated by the contributors of the present work, the short-term follow-up period along with the heterogeneity of the applied behavioral interventions could justify this observation.

A range of complementary life course strategies has been suggested in the literature at individual, population or health care system level, with a dual purpose; to improve cardiac health of individuals with unhealthy lifestyle and clinical comorbidities in their medical history and to support the retention of an optimal risk factor profile in apparently healthy individuals (2). The USPSTF addresses a specific target group (i.e., low CVD risk subjects) providing a guideline to primary health care professionals on an individual-centered basis; "to individualize the decision to offer or refer low CVD risk adults to behavioral counseling to promote a bealthful diet and physical activity" (Grade C).

According to World Health Organization, $80 \%$ of CVD morbidity and mortality rates would be prevented on the grounds of adherence to health-related behaviors such as optimal dietary habits and physical activity status.
However, this should not be the finishing point. In the present and other relevant systematic reviews performed by USPSTF, only modest short-term benefits were highlighted and solely in case of intermediate health outcomes (8). Thereby, translating the knowledge into actual practice is always remaining a complex issue in CVD prevention lifestyle-centered strategies to achieve outcomes with a high clinical importance (9). To meet the challenge of lifestyle modification, the nature of the whole applied intervention seems detrimental. In this context, efficient psychological and behavioral theoretical principles should be taken into serious consideration (10). Most importantly, the appropriate-for-the target group delivery mode, schedule, nature of counseling and intensity of the whole procedure should be selected with consciousness $(10,11)$. The readiness to change is another fundamental parameter in lifestyle interventions which can determine their progress and efficiency; also, implied in the present guideline provided by the USPSTF. This is the reason for which patients at high-risk with clinical symptoms and signs are more likely to benefit from behavioral counseling (11). Hence, when it comes to lifestyle interventions applied to apparently healthy individuals, application of motivational techniques is strongly suggested to achieve a long-term compliance $(12,13)$.

Behavioral counseling of healthy nutrition and physical activity seems modestly beneficial in primary CVD prevention. Therefore, it is justifiably recommended in primary health care professionals. However, the strategies and techniques through which this recommendation will be put into the real-world setting needs a better clarification. In a global society with finite healthcare resources, the effective integration of low cost individual-centered lifestyle modification approaches seems a compelling strategy to meet the challenges in the CVD prevention spectrum. Primary health care practitioners should be encouraged to incorporate them in their daily clinical practice. Consequently, the need for a new generation of studies with tailor-made behavioral lifestyle counseling is imperative to achieve more strong, tangible and long-term outcomes.

\section{Acknowledgments}

None.

\section{Footnote}

Conflicts of Interest: The authors have no conflicts of interest 
to declare.

\section{References}

1. Mensah GA, Wei GS, Sorlie PD, et al. Decline in Cardiovascular Mortality: Possible Causes and Implications. Circ Res 2017;120:366-80.

2. Benjamin EJ, Blaha MJ, Chiuve SE, et al. Heart Disease and Stroke Statistics-2017 Update: A Report From the American Heart Association. Circulation 2017;135:e146-603.

3. U.S. Department of Health and Human Services. Centers for Disease Control and Prevention. National Center for Health Statistics. Health, United States, 2015. With Special Feature on Racial and Ethnic Health disparities 2016. Available online: https://www.cdc.gov/nchs/data/ hus/hus15.pdf

4. Yusuf S, Hawken S, Ounpuu S, et al. Effect of potentially modifiable risk factors associated with myocardial infarction in 52 countries (the INTERHEART study): case-control study. Lancet 2004;364:937-52.

5. Koloverou E, Esposito K, Giugliano D, et al. The effect of Mediterranean diet on the development of type 2 diabetes mellitus: a meta-analysis of 10 prospective studies and 136,846 participants. Metabolism 2014;63:903-11.

6. Esposito K, Kastorini CM, Panagiotakos DB, et al. Mediterranean diet and metabolic syndrome: an updated systematic review. Rev Endocr Metab Disord 2013;14:255-63.

7. Huo R, Du T, Xu Y, et al. Effects of Mediterranean-style diet on glycemic control, weight loss and cardiovascular

Cite this article as: Panagiotakos DB, Kouvari M. Behavioral healthy nutrition and physical activity counseling in cardiovascular disease prevention; where we are now? HepatoBiliary Surg Nutr 2019;8(5):534-536. doi: 10.21037/ hbsn.2018.11.16 risk factors among type 2 diabetes individuals: a metaanalysis. Eur J Clin Nutr 2015;69:1200-8.

8. Lin JS, O'Connor E, Evans CV, et al. Behavioral counseling to promote a healthy lifestyle in persons with cardiovascular risk factors: a systematic review for the U.S. Preventive Services Task Force. Ann Intern Med 2014;161:568-78.

9. Ravera A, Carubelli V, Sciatti E, et al. Nutrition and Cardiovascular Disease: Finding the Perfect Recipe for Cardiovascular Health. Nutrients 2016;8. doi: 10.3390/ nu8060363.

10. Bully P, Sánchez Á, Zabaleta-del-Olmo E, et al. Evidence from interventions based on theoretical models for lifestyle modification (physical activity, diet, alcohol and tobacco use) in primary care settings: A systematic review. Prev Med 2015;76 Suppl:S76-93.

11. Zabaleta-del-Olmo E, Bolibar B, García-Ortíz L, et al. Building interventions in primary health care for long-term effectiveness in health promotion and disease prevention. A focus on complex and multi-risk interventions. Prev Med 2015;76 Suppl:S1-4.

12. Maderuelo-Fernandez JA, Recio-Rodríguez JI, PatinoAlonso MC, et al. Effectiveness of interventions applicable to primary health care settings to promote Mediterranean diet or healthy eating adherence in adults: A systematic review. Prev Med 2015;76 Suppl:S39-55.

13. Livia B, Elisa R, Claudia R, et al. Stage of Change and Motivation to a Healthier Lifestyle before and after an Intensive Lifestyle Intervention. J Obes 2016;2016:6421265. 